\title{
Lower Bounds of the Smallest Singular Value of Matrices
}

\author{
Liao Ping
}

Correspondence: SiChuan vocational and technical college, Faculty of Education, SuiNing,SiChuan, PR China

Received: July 9, 2021 Accepted: August 9, 2021 Online Published: August 24, 2021

doi:10.5539/jmr.v13n5p1 URL: https://doi.org/10.5539/jmr.v13n5p1

\section{Abstract}

In this paper, we get a lower bound of the smallest singular value of an arbitrarily matrix $A$ by the trace of $H(A)$ and the Euclidean norm of $H(A)$, where $H(A)$ is Hermitian part of $A$, numerical examples show the effectiveness of our results.

Keywords: smallest singular value, lower bounds, matrices

AMS Classfication: 15A42,15A48

\section{Introduction}

Let $A=\left[a_{i j}\right]$ be an $n \times m$ matrix and $A^{*}$ be the conjugate transpose of $A$, the singular values of $A$ are eigenvalues of $\left(A A^{*}\right)^{\frac{1}{2}}$ and denoted as $\sigma_{1} \geq \sigma_{2} \geq \cdots \geq \sigma_{n} \geq 0$. In this paper, we will focus on the smallest singular value $\sigma_{n}$, which is important in assessing numerical calculations (M. Marcus, H.Minc, 1992) and the singularity of a matrix. Without loss of generality, we only consider $n \times n$ matrices in the following discussing. Let

$$
P_{i}(A)=\sum_{k \neq i} a_{i k}, i=1,2, \ldots, n,
$$

and

$$
Q_{i}(A)=\sum_{k \neq i} a_{k i}, i=1,2, \ldots, n .
$$

If $a_{i i} \geq P_{i}(A)$ and $a_{i i} \geq Q_{i}(A), i=1, \cdots, n$, In (Varah J M., 1975), the following lower bound is proposed

$$
\sigma_{n} \geq\left(\min \left(\left|a_{i i}\right|-P_{i}(A)\right) \min \left(\left|a_{i i}\right|-Q_{i}(A)\right)\right)^{\frac{1}{2}} .
$$

Denote $H(A)=\left(A+A^{*}\right) / 2$, this lower bound was improved by Johnson in (Johnson C R.,1989), that is

$$
\sigma_{n} \geq \min \left\{\operatorname{Rea}_{i i}-P_{i}(H(A))\right\}
$$

and

$$
\sigma_{n} \geq \min \left\{\left|a_{i i}\right|-P_{i}(H(D A))\right\},
$$

where $D$ is a diagonal matrix which transform $a_{i i}$ to $\left|a_{i i}\right|, i=1, \cdots, n$. These lower bounds are simple and easy to compute, but require the modules of all the diagonal entries be big enough, there are also some other results in (Huang $\mathrm{T}$ Z, You Z Y.,1994)) and (Johnson C R, Tomasz Szulc.,1998). In this paper, we get a different lowet bound which depend on the trace of matrix $A$ and the Euclidean norm of $H(A)$. And this new lower bound is not comparable with (1) or $(2,3)$.

\section{Main Results}

To get our results, we need the following lemmas.

lemma 1 Let $A=\left[a_{i j}\right]$ be a complex matrix of order $n, x$ is the right eigenvector of $\sigma_{n}, H(A)=\left(A+A^{*}\right) / 2$, then

$$
\sigma_{n} \geq\left|x^{*} H(A) x\right|
$$

Proof For an arbitrarily real number $t$, Let $M=A-t I$, then $M M^{*}=A A^{*}-t\left(A+A^{*}\right)+t^{2} I$, that is

$$
A A^{*}=M M^{*}+t\left(A+A^{*}\right)-t^{2} I,
$$

Note that $M M^{*}$ is semi positive definite and $x$ is the right eigenvector of singular value of $\sigma_{n}$, then we get 


$$
\sigma_{n}^{2}(A)=x^{*} A A^{*} x=x^{*} M M^{*} x+t \cdot x^{*}\left(A+A^{*}\right) x-t^{2} \geq t \cdot x^{*}\left(A+A^{*}\right) x-t^{2} .
$$

Let $t=x^{*}\left(A+A^{*}\right) x / 2$, one can get

$$
\sigma_{n}^{2}(A) \geq \frac{x^{*}\left(A+A^{*}\right) x}{4}=\left|x^{*} H(A) x\right|^{2} .
$$

that is $\sigma_{n} \geq\left|x^{*} H(A) x\right|$, and the proof is complete.

lemma 2 (Gu Y X., 1994) Let $A=\left[a_{i j}\right]$ be a complex matrix of order $n, \lambda$ is an arbitrarily eigenvalue of $A$, then

$$
\left|\lambda-\frac{\operatorname{trA}}{n}\right| \leq \sqrt{\frac{n-1}{n}\left(\|A\|^{2}-\frac{|t r A|^{2}}{n}\right)}
$$

Now we are ready to get our lower bounds of $\sigma_{n}$.

Theorem 1 Let $A=\left[a_{i j}\right]$ be a complex matrix of order $n$ and $\sigma_{n}$ be its smallest singular value, denote $H(A)=\left(A+A^{*}\right) / 2$, then

$$
\sigma_{n}(A) \geq\left|\frac{\operatorname{tr} H(A)}{n}\right|-\sqrt{\frac{n-1}{n}\left(\|H(A)\|^{2}-\frac{|\operatorname{trH}(A)|^{2}}{n}\right)} .
$$

Proof Let $\lambda_{n}$ be the smallest eigenvalue of $H(A)$ and $\lambda_{1}$ be the biggest eigenvalue of $H(A)$.

Case 1, if

$$
\frac{\operatorname{tr} H(A)}{n}-\sqrt{\frac{n-1}{n}\left(\|H(A)\|^{2}-\frac{|\operatorname{trH}(A)|^{2}}{n}\right)} \geq 0,
$$

then by lemma 2 we get

$$
\left.\lambda_{n} \geq \frac{\operatorname{tr} H(A)}{n}-\sqrt{\frac{n-1}{n}\left(\|H(A)\|^{2}-\frac{|\operatorname{trH}(A)|^{2}}{n}\right.}\right) \geq 0,
$$

which means the matrix $H(A)$ is positive semidefinite.

and by lemma 1 , we get

$$
\sigma_{n} \geq\left|x^{*} H(A) x\right| \geq \lambda_{n}
$$

Then we get

$$
\sigma_{n} \geq \lambda_{n} \geq \frac{\operatorname{tr} H(A)}{n}-\sqrt{\frac{n-1}{n}\left(\|H(A)\|^{2}-\frac{|\operatorname{trH}(A)|^{2}}{n}\right)} .
$$

Case 2, if

$$
\left.\frac{\operatorname{tr} H(A)}{n}+\sqrt{\frac{n-1}{n}\left(\|H(A)\|^{2}-\frac{|\operatorname{trH}(A)|^{2}}{n}\right.}\right) \leq 0,
$$

then by lemma 2, the biggest eigenvalue of $H(A)$,e.g $\lambda_{1}$ satisfies

$$
\lambda_{1} \leq \frac{\operatorname{tr} H(A)}{n}+\sqrt{\frac{n-1}{n}\left(\|H(A)\|^{2}-\frac{|\operatorname{trH}(A)|^{2}}{n}\right)} \leq 0 .
$$

This tells us that the matrix $H(A)$ is negative semidefinite, and by lemma 1, we get

$$
\sigma_{n} \geq\left|x^{*} H(A) x\right| \geq\left|\lambda_{1}\right|,
$$

then we have

$$
\sigma_{n} \geq\left|\lambda_{1}\right| \geq-\frac{\operatorname{tr} H(A)}{n}-\sqrt{\frac{n-1}{n}\left(\|H(A)\|^{2}-\frac{|\operatorname{trH}(A)|^{2}}{n}\right)} .
$$

In conclusion,

$$
\sigma_{n} \geq\left|\lambda_{1}\right| \geq\left|\frac{\operatorname{tr} H(A)}{n}\right|-\sqrt{\frac{n-1}{n}\left(\|H(A)\|^{2}-\frac{|\operatorname{trH}(A)|^{2}}{n}\right)}
$$

the proof is complete. 
Now we give an example to show the effectiveness of our result.

\section{Example 1 Suppose}

$$
A=\left(\begin{array}{lll}
1 & 0 & 1 \\
0 & 8 & 1 \\
1 & 1 & 9
\end{array}\right)
$$

By the use of (1) or (2), we just get $\sigma_{n}>0$. While if we use (6), we can get $\sigma_{n}>0.71$. In fact, we can see that the lower bound in (6) is based on the trace of $A$, which means all the diagonal entries are considered together, if the module of the trace of $A$ is big enough, one can get a positive lower bound by (6). This is different from the lower bounds in (1) or (2), which require every diagonal entry must be "big" enough.

And we have to point out that this do not mean (6) is always better than (1) or (2), consider the following example.

\section{Example 2 Suppose}

$$
A=\left(\begin{array}{cc}
2 & 1.9 \\
1.9 & -2
\end{array}\right) \text {, }
$$

By (1), we get $\sigma_{n}>0.1$,by (2) we get $\sigma_{n}>0.1$, while by (6) we just get trivial lower bound. So these bounds are not comparable.

Under this situation,we can use a similar method to transform the diagonal entries $a_{i i}$ to $\left|a_{i i}\right|$ like (Johnson C R.,1989), and by theorem 1 , we get can the following lower bound.

Theorem 2 Let $A=\left[a_{i j}\right]$ be a complex matrix of order $n$ and $\sigma_{n}$ be its smallest singular value, denote $H(A)=\left(A+A^{*}\right) / 2$, then

$$
\sigma_{n}(A) \geq\left|\frac{\operatorname{tr} H(D A)}{n}\right|-\sqrt{\frac{n-1}{n}\left(\|H(D A)\|^{2}-\frac{|\operatorname{tr} H(D A)|^{2}}{n}\right)},
$$

where $D$ is a diagonal matrix and the diagonal entries of $D A$ are $\left|a_{i i}\right|, i=1, \cdots, n$.

The proof is similar to (Johnson C R.,1989), we omit it here.

At last, we propose a determination about the singularity of a matrix by (6). That is

corollary 1 Let $A=\left[a_{i j}\right]$ be a complex matrix of order $n$, denote $H(A)=\left(A+A^{*}\right) / 2$, if $|\operatorname{tr} H(A)|>\sqrt{n-1}\|H(A)\|$, then $A$ is nonsingular.

Proof In (6), let

$$
\left|\frac{\operatorname{tr} H(A)}{n}\right|-\sqrt{\frac{n-1}{n}\left(\|H(A)\|^{2}-\frac{|\operatorname{trH}(A)|^{2}}{n}\right)} \geq 0 .
$$

that is

$$
\left|\frac{\operatorname{tr} H(A)}{n}\right|^{2} \geq \frac{n-1}{n}\left(\|H(A)\|^{2}-\frac{|\operatorname{tr} H(A)|^{2}}{n}\right) .
$$

simplify this inequality, we can get

$$
|\operatorname{tr} H(A)|>\sqrt{n-1}|| H(A) \| .
$$

then $\sigma_{n}>0$, which indicates that the matrix $A$ is nonsingular.

Similarly, by (7), we can also have

corollary 2 Let $A=\left[a_{i j}\right]$ be a complex matrix of order $n$, denote $H(A)=\left(A+A^{*}\right) / 2$, and $D$ is the same as theorem 2 . If $|\operatorname{tr} H(D A)|>\sqrt{n-1}\|H(D A)\|$, then $A$ is nonsingular.

\section{Conclusion}

In this paper,we get a lower bound of the smallest singular value of an arbitrarily matrix $A$ by $\operatorname{tr} H(A)$ and the Euclidean norm of $H(A)$, where $H(A)=\left(A+A^{*}\right) / 2$ is the Hermitian part of $A$. This bound is different from the classical results which require every diagonal entry must be "big" enough. Numerical examples show the effectiveness of our results.

\section{References}

Marcus, M., \& Minc, H. (1992). A Survey of Matrix Theory and Matrix Inequalities. Dover Publications, New York.

Varah, J. M. (1975). A lower bound for the smallest singular value. Linear Algebra Appl., 11, 3-5. https://doi.org/10.1016/00243795(75)90112-3 
Johnson, C. R. (1989). A Gersgorin-type lower bound for the smallest singular value. Linear Algebra Appl., 112, 1-7. https://doi.org/10.1016/0024-3795(89)90583-1

Huang, T. Z., \& You, Z. Y. (1994). A lower bound for the smallest singular value. Chinese Journal of engineering mathematics, 11, 110-112.

Johnson, C. R., \& Tomasz, S. (1998). Further lower bounds for the smallest singular value. Linear Algebra Appl., 272, 169-179. https://doi.org/10.1016/S0024-3795(97)00330-3

Gu, Y. X. (1994). The distribution of eigenvules of a matrix. ACTA Mathematicae Applicatae Sinica, 4, 501-511.

\section{Copyrights}

Copyright for this article is retained by the author(s), with first publication rights granted to the journal.

This is an open-access article distributed under the terms and conditions of the Creative Commons Attribution license (http://creativecommons.org/licenses/by/4.0/). 